\title{
Brain amyloid accumulation in senescent rats with kaolin-induced hydrocephalus
}

\author{
PM Klinge*, T Brinker, A Samii and GD Silverberg
}

\author{
Address: International Neuroscience Institute Hannover, Alexis-Carrel-Str. 4, 30625 Hannover, Germany \\ Email: PM Klinge* - klinge@ini-hannover.de \\ * Corresponding author
}

from 49th Annual Meeting of the Society for Research into Hydrocephalus and Spina Bifida

Barcelona, Spain, 29 June - 2 July 2005

Published: 30 December 2005

Cerebrospinal Fluid Research 2005, 2(SuppI I):S32 doi:I0.II86/I743-8454-2-SI-S32

\section{Background}

NPH patients have a high rate of Alzheimer's disease (AD) on cortical biopsy. $30-50 \%$ of shunted NPH patients show amyloid $(A \beta)$ plaques and neurofibrillary tangles. It is postulated that $A \beta$ accumulates in AD and NPH due to decreased $A \beta$ clearance via CSF and blood-brain barrier (BBB). The present study investigates $A \beta$ accumulation and $A \beta$ transport in aged hydrocephalic rat brains.

\section{Materials and methods}

Kaolin-hydrocephalus was induced in senescent (12 months) SD-rats. Untreated age- matched rats served as controls. $A \beta$ accumulation was investigated by specific $A \beta(1-40)$ and $A \beta(1-42)$ antibody immunohistochemistry performed 2 weeks (short-term), 6 and 10 weeks (longterm) after hydrocephalus induction. Each group consisted of five animals. Also, specific BBB A $\beta$ receptors were labelled: LRP-1, which transports $A \beta$ from the interstitial fluid (ISF) into the plasma, and RAGE, which transports $\mathrm{A} \beta$ from the plasma into the ISF. Both receptors are located on the capillary endothelium.

\section{Results}

After 2 weeks of hydrocephalus, both $A \beta 42$ and $A \beta 40$ showed increased staining of the arachnoid and ependyma compared to controls. Cortical and hippocampal CA3 pyramidal neurons displayed A $\beta 42$ cytoplasmic staining in some animals. At 6 weeks, cortical and hippocampal endothelial and perivascular $A \beta 42$ and 40 accumulations were observed, most prominently with $A \beta 42$. Importantly, interstitial $A \beta 42$ and $A \beta 40$ accumulations were observed, and periventricular plaque-like formations were found in all animals. At 10 weeks, the observed plaque-like formations were increased, whereas cortical perivascular accumulations varied and were either increased or identical to the 6 weeks animals. LRP-receptor staining was decreased in cortical and subcortical vessels at two weeks. However, the decrease was most prominent after 6 weeks. After 10 weeks, LRP-1 receptor staining was restricted to large dilated capillary vessels. RAGE receptor staining showed diametrically opposite changes to those seen for the LRP-1 receptor.

\section{Conclusion}

In a rat model of chronic hydrocephalus, perivascular, interstitial and periventricular accumulations of $A \beta 42$ and 40 , both of which play a major role in $\mathrm{AD}$-plaque formation, are observed, with $A \beta$ staining increasing the longer hydrocephalus exists. BBB receptor staining indicates impaired $A \beta$ clearance from the ISF into the plasma. These preliminary studies indicate that $A \beta$ accumulation in hydrocephalus is, in part, due to a failure of brain amyloid clearance as it is in $\mathrm{AD}$. Reduced CSF turnover seen in $\mathrm{AD}$, $\mathrm{NPH}$ and rat kaolin-hydrocephalus, and reduced $\mathrm{A} \beta$ net transport at the BBB appear to be involved. Perivascular $\mathrm{A} \beta$ accumulation, known to be a potent vasoconstrictor, may also play a role in the white-matter ischaemia seen in both human NPH and in rat chronic hydrocephalus. 